$(1.26(1.03,1.53))$, and greater comorbidity $(1.05(1.00,1.09))$, higher levels of pain $(1.11(1.04,1.18))$ and poorer balance $(1.25(1.05,1.49))$ in men. Of these, only the relationships between pain, balance and comorbidity and falls risk differed significantly $(\mathrm{p}<0.05)$ by gender according to interaction tests. Conclusion Older age was associated with increased risk of incident falls in both sexes. However, associations regarding pain, balance and comorbidity differed by gender. Strengths of the study include the large sample size and the fact that it is representative of the community-dwelling English population aged 60 years and over. However, one potential weakness is that there was no definition in the questionnaire of what constituted a fall. This research will inform the development of interventions to reduce the risk of falls among older people.

\section{P60 ALCOHOL CONSUMPTION AND RISK OF INCIDENT FRAILTY: THE ENGLISH LONGITUDINAL STUDY OF AGEING}

${ }^{1} \mathrm{G}$ Kojima*, ${ }^{2} \mathrm{~S}$ Jivraj, ${ }^{1} \mathrm{~S}$ lliffe, ${ }^{1} \mathrm{~A}$ Liljas, ${ }^{1} \mathrm{~K}$ Walters. ${ }^{1}$ Department of Primary Care and Population Health, University College London, London, UK; ${ }^{2}$ Department of Epidemiology and Public Health, University College London, London, UK

\subsection{6/jech-2018-SSMabstracts.183}

Background Frailty is recognised as an important public health concern among older people due to its detrimental consequences on health. Alcohol consumption may be a plausible risk factor for frailty. There is only limited evidence in the literature on association between alcohol and frailty, which is still controversial. Therefore we investigated the relationship between alcohol consumption and the risk of incident frailty.

Methods A total of 2544 community-dwelling older people aged $\geq 60$ years from the English Longitudinal Study of Ageing were followed for incident frailty over four years according to alcohol consumption. Frailty was defined using Fried frailty phenotype criteria with slight modification according to data availability. Questionnaire data on alcohol consumption was grouped into non-drinkers, >0-7 units/week, >7-14 units/week, >14-21 units/week, >21 units/week. Multivariable logistic regression models were used to examine incident frailty risk.

Results Compared with those drinking $>0-7$ units per week (reference), those drinking $>21$ units per week were less likely to develop frailty (Odds ratio $(\mathrm{OR})=0.45,95 \%$ confidence interval $(\mathrm{CI})=0.27-0.73, \mathrm{p}<0.001)$ in the unadjusted model, however the association became non-significant in the fully adjusted model controlling for age, gender, smoking, education and wealth $(\mathrm{OR}=0.65,95 \%$ CI 0.38 to $1.11, \mathrm{p}=0.12)$. There was no significant difference in incident frailty risk in those drinking $>7-14$ and $>14-21$ units per week compared to $>0-7$ units per week in any models. Non-drinkers had worse health profile and higher incident frailty risk than drinkers (fully adjusted $\mathrm{OR}=1.63$, 95\% CI 1.07 to 2.48, $\mathrm{p}=0.02)$.

Conclusion In this cohort non-drinkers had a significantly higher risk of developing frailty compared to light drinkers (>0-7 units per week). No significant associations were observed among drinkers. The true association between alcohol and frailty may have been affected by a 'sick-quitter' effect (i.e. drinkers who are ill health tend to reduce or cease alcohol use), which should be addressed in future research.

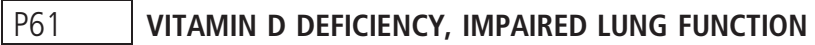 AND TOTAL MORTALITY IN OLDER MEN: THE BRITISH REGIONAL HEART STUDY}

${ }^{1}$ SG Wannamethee*, ${ }^{2}$ Paul Welsh, ${ }^{1} \mathrm{O}$ Papacosta, ${ }^{1} \mathrm{LT}$ Lennon, ${ }^{3} \mathrm{PH}$ Whincup. ${ }^{1}$ Department Primary Care and Population Health, University College London, London, UK; ${ }^{2}$ Institute of Cardiovascular and Medical Sciences, BHF Glasgow Cardiovascular Research Centre, University of Glasgow, Glasgow, UK; ${ }^{3}$ Population Health Research Institute, St George's University of London, London, UK

\subsection{6/jech-2018-SSMabstracts. 184}

Background Chronic obstructive lung disease (COPD) is a common chronic inflammatory disease in the elderly and is a major cause of morbidity and mortality. Much attention has focussed on identifying factors which may influence prognosis and mortality in these patients. Although vitamin D deficiency is traditionally known for its role in bone health, there is growing interest in the role of vitamin $\mathrm{D}$ in COPD. Observational studies have found Vitamin D deficiency to be highly prevalent in patients with COPD. We have examined the association between vitamin D deficiency (ascertained from measuring the circulating 25-dehydroxyvitamin D [25OHD] metabolite) and lung function impairment and assessed whether vitamin D deficiency is related to long term mortality in those with impaired lung function.

Methods Prospective study of 3575 men aged 60-79 years with no prevalent HF followed up for 15 years, in whom there were 1937 deaths (80 COPD deaths). The Global Initiative on Obstructive Lung diseases (GOLD) spirometry criteria was used to define airway obstruction. Vitamin D deficiency was defined as serum $25 \mathrm{OHD}$ levels $<10 \mathrm{ng} / \mathrm{ml}$; insufficiency as $25 \mathrm{OHD} 10-19 \mathrm{ng} / \mathrm{mL}$; normal as $25 \mathrm{OHD}>20 \mathrm{ng} / \mathrm{mL}$.

Results Vitamin D deficiency was associated with moderate COPD (FEV/FVC $<70 \%$ and FEV1 50\%-80\%) and severe COPD (FEV/FVC $<70 \%$ and FEV1 <50\%) but not with mild COPD (FEV/FVC $<70 \%$ and FEV1 $>80 \%$ ) or restrictive lung disease (FEV1/FVC $>70 \%$ and FVC $<80 \%)$. Vitamin D deficiency was associated with significantly increased risk of total and COPD mortality in all men except those with severe COPD after adjustment for confounders. The adjusted HRs (95\% CI) for total mortality comparing levels of 25OHD $<10 \mathrm{ng} / \mathrm{mL}$ to $25 \mathrm{OHD}>20 \mathrm{ng} / \mathrm{ml}$ were $1.38(1.10,1.75)$, $1.62(1.22,2.15), 1.93(1.32,2.80)$ and $1.34(0.78,2.31)$ for those with none/mild COPD, restrictive lung function, moderate COPD and severe COPD respectively. The corresponding HRs $(95 \% \mathrm{CI})$ for COPD deaths were 14.3 (2.45, 83.23), $5.10(0.96,27.06), 3.55(1.12,11.22)$ and $0.81(0.25,2.57)$ for the 4 groups respectively.

Conclusion Our findings suggest that older adults with vitamin D deficiency $(<10 \mathrm{ng} / \mathrm{ml})$ and earlier stages of lung impairment may potentially benefit from vitamin D supplementation. Intervention trials in older people with mild or moderate impaired lung function are needed to confirm whether increasing vitamin $\mathrm{D}$ levels through supplements in those with vitamin D deficiency will reduce both risk of COPD deaths and overall mortality. 